\title{
A Note on Numerical Solution of Classical Darboux Problem
}

\author{
Kotapally Kumar ${ }^{1}$ and Ram Jiwari² \\ ${ }^{1}$ IIT Roorkee \\ ${ }^{2}$ Indian Institute of Technology Roorkee
}

June 26, 2020

\begin{abstract}
Recently, many authors studied the numerical solution of the classical Darboux problem in its integral form via a two-dimensional nonlinear Volterra-Fredholm integral equation. In the present article, a numerical technique based on the Chebyshev wavelet is proposed to solve the Darboux problem directly without converting into a nonlinear Volterra-Fredholm integral equation. The proposed technique is different from the techniques discussed in $[1,2,4,7,11,16,17,18,19]$. The proposed approach produces higher accuracy than its counterpart techniques. The proposed scheme illustrated with suitable examples to show the advantages in terms of its accuracy with lesser grid size.
\end{abstract}

\section{Hosted file}

Harish_Jiwari.pdf available at https://authorea.com/users/337125/articles/462773-a-note-onnumerical-solution-of-classical-darboux-problem

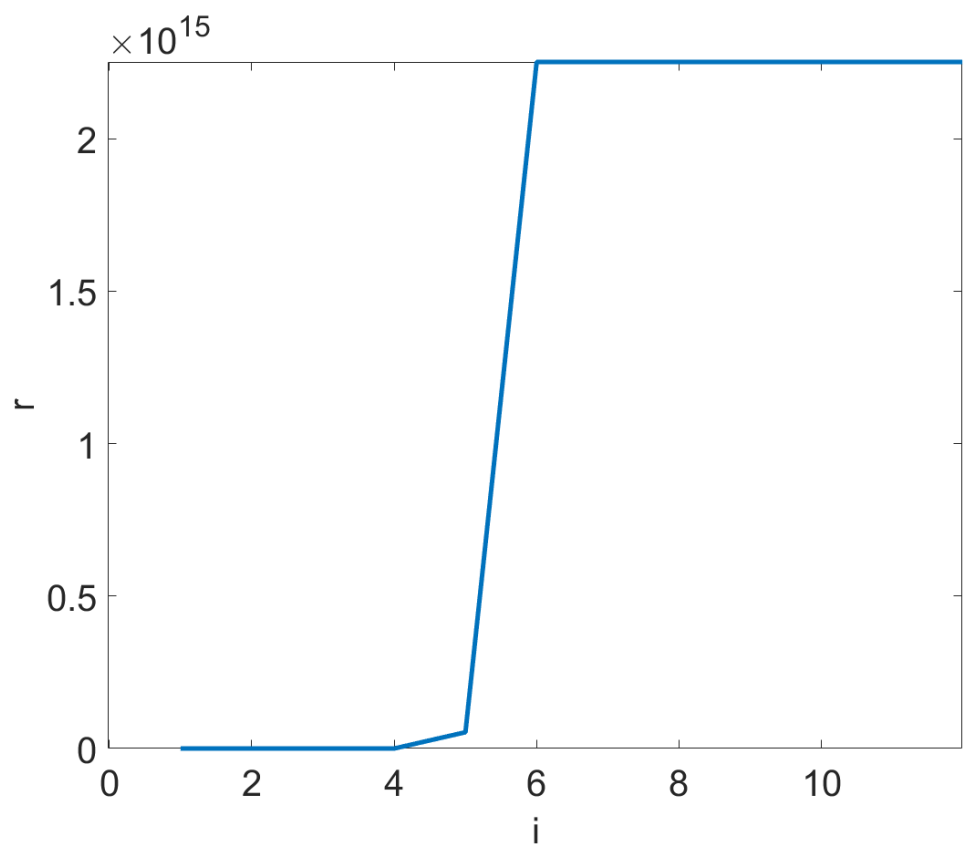



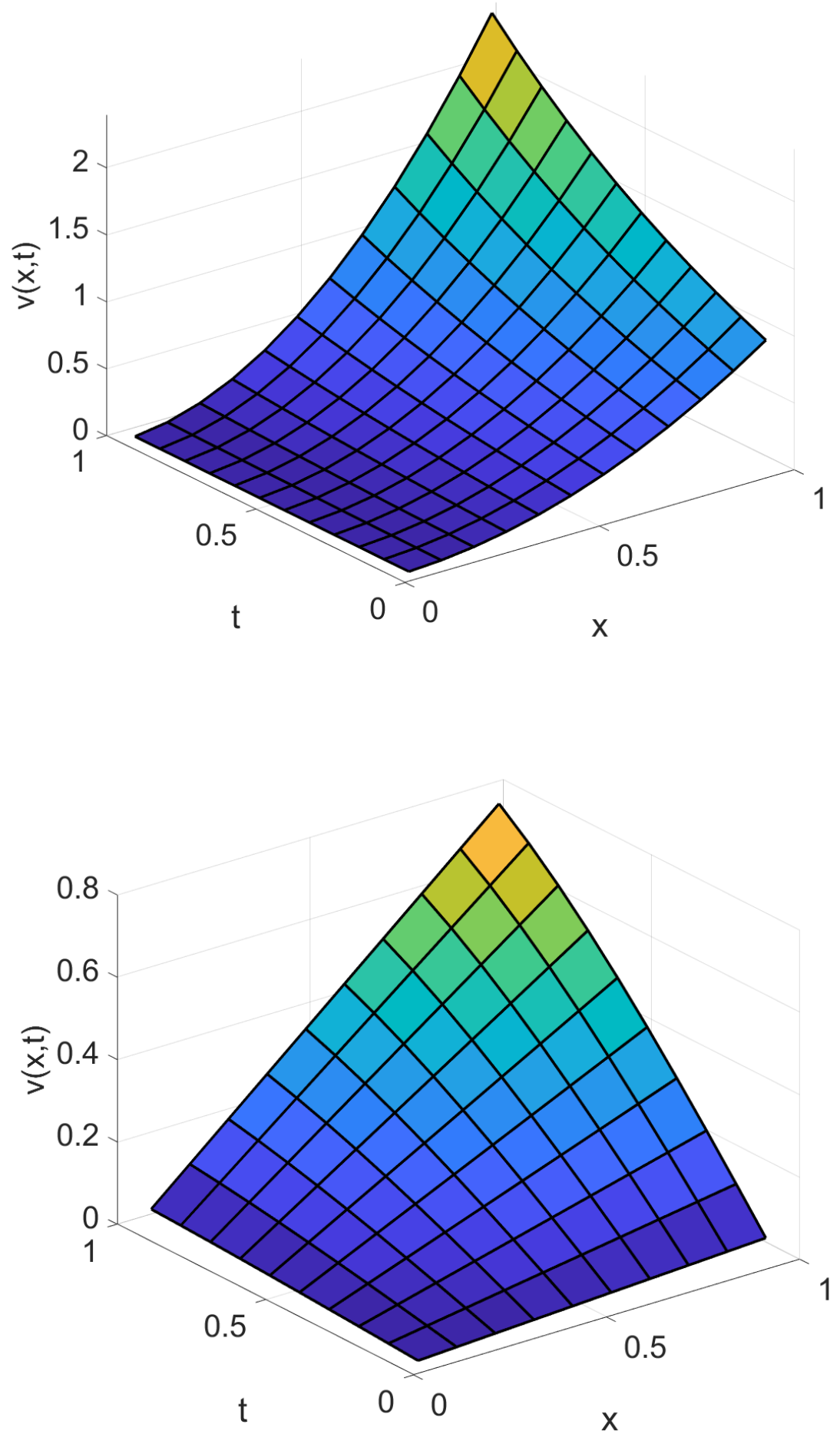

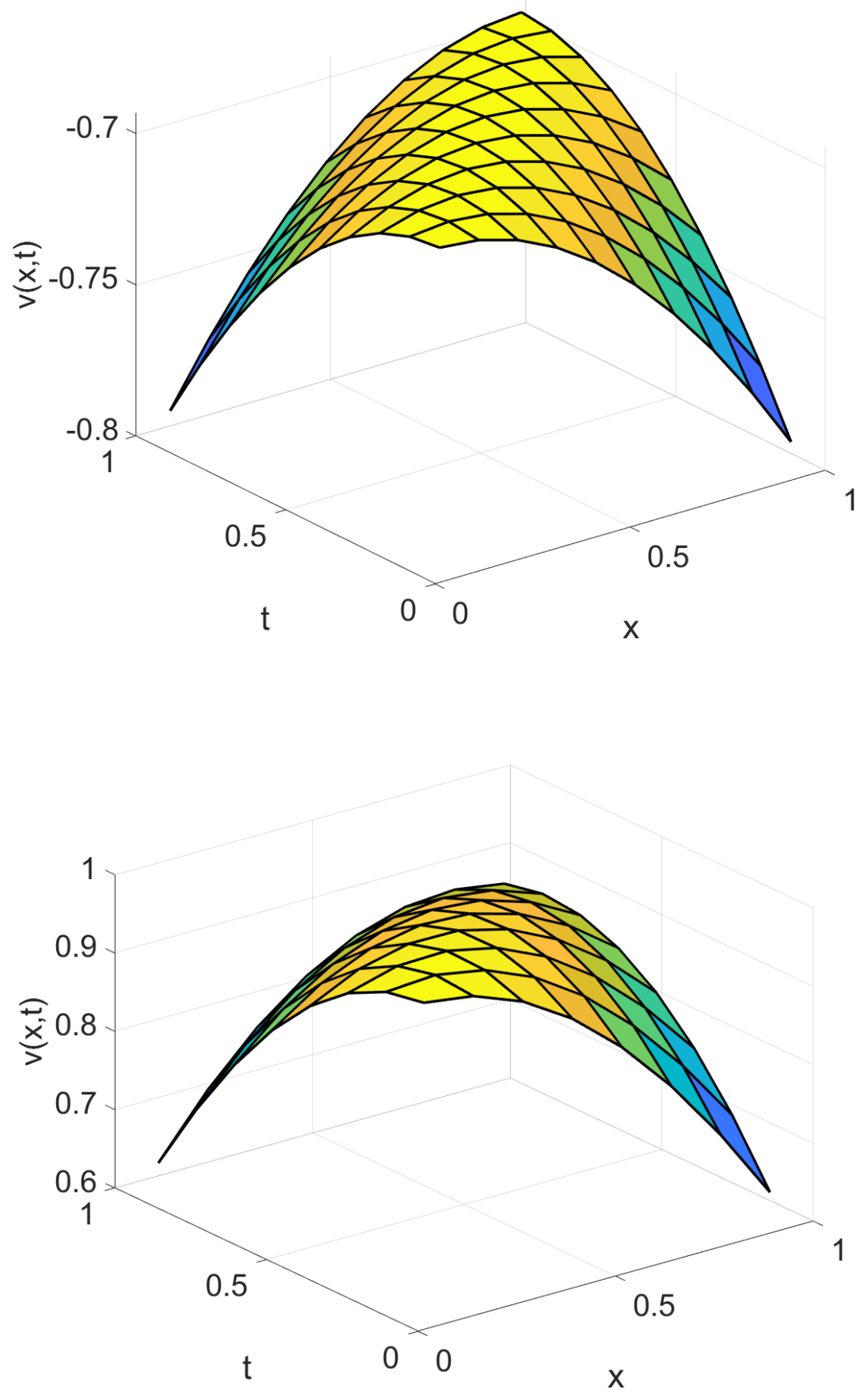